\title{
Periorbital Soft Tissue Trauma
}

\author{
Young Seol, MD ${ }^{1}$ Monica Dweck, MD² \\ 1 Department of Ophthalmology, New York Eye and Ear Infirmary at \\ Mount Sinai, New York, New York \\ 2 Department of Ophthalmology and Medical Education, Icahn School \\ of Medicine at Mount Sinai, New York, New York
}

Address for correspondence Monica Dweck, MD, Department of Ophthalmology and Medical Education, Icahn School of Medicine at Mount Sinai, 111 Lawrence St, Apt 40 H, Brooklyn, NY 11201 (e-mail: Mdweck2@gmail.com).

Facial Plast Surg 2021;37:463-472.

\author{
Abstract \\ Keywords \\ - soft tissue injuries \\ - periorbital trauma \\ - oculoplastic repair \\ - eyelid trauma \\ - canalicular \\ lacerations
}

Eyelid trauma occurs across a broad spectrum of pathology, ranging from simple periorbital lacerations to severe, vision-threatening injuries requiring expert oculoplastic consultation. Any injury, no matter how benign, is also inherently cosmetically sensitive, further adding to the reconstructive challenge. In this review, we discuss the anatomy of the eyelid and develop an understanding of evaluating for signs of more serious, potentially occult, trauma. A framework is developed for approaching the patient with periorbital trauma to assess for injury and triage necessary treatments. Damage to the lacrimal drainage system, which can be particularly difficult to detect and repair, is specifically emphasized and explored.
When approaching trauma to the soft tissues of the face, few areas are as functionally and cosmetically sensitive as the eyelids. In this review, we will develop an understanding of the anatomy of this delicate structure and how it informs general principles for repair. We pay special attention to the reconstruction of the canaliculi, which may be injured in obvious and nontrivial ways: how to assess for the presence of damage and specific techniques in repairing it.

Although a wide variety of surgeons develop an expertise in treating facial trauma, the oculoplastic surgeon remains uniquely qualified in understanding the interplay of cosmesis, anatomy, and functionality with respect to the eyeball as it relates to the repair of eyelid trauma. Incomplete or poor repair of the eyelids can cause significant decrease in quality of life, such as ectropion or entropion, trichiasis, or epiphora in addition to cosmetic dysfunction that may be socially significant. In severe cases, untreated or mistreated eyelid trauma may lead to vision-threatening sequalae such as exposure keratopathy and corneal ulcers.

\section{Anatomy}

Any approach to the oculoplastic repair of orbital soft tissue injuries requires a comprehensive understanding of the anatomy. Attention must be paid during reconstruction to restoration of functional status, which is often a separate and more difficult objective to achieve than simple anatomical outcomes. We begin with a discussion of the major eyelid structures that enable these functions before delving deeper into a discussion of the canalicular system and other anterior structures relevant to soft tissue injuries. A detailed analysis of the globe, bony orbit, and posterior orbital contents is certainly necessary for the ophthalmic consultant but is beyond the scope of this review (-Fig. $\mathbf{1}$ ).

It helps to first understand the ways in which the upper and lower eyelids are similar before delving into a comparison of their differences. Both eyelids have two muscles that contribute to opening or retracting the lids: superiorly the levator palpebrae superioris muscle and Muller's muscle, also known as the superior tarsal muscle, and inferiorly the capsulopalpebral fascia and inferior tarsal muscle. ${ }^{1}$ The upper and lower eyelid retractors are innervated by the third cranial nerve and sympathetic fibers, respectively. Somatic control of the levator accounts for the majority of eyelid movement, with normal excursion in the range of 13 to $17 \mathrm{~mm}$. Muller's muscle provides approximately $2 \mathrm{~mm}$ of retraction. $^{1-3}$

In comparison to the upper eyelid retractors, the lower eyelid retractors are less developed. The chief role of the lower eyelid retractors is to move the lower eyelid out of the way during downgaze, for instance during reading or walking. This functional purpose then also explains the published online March 3, 2021
Issue Theme Facial Soft Tissue Injury and Healing; Guest Editor: Sydney C. Butts, MD, FACS (c) 2021. Thieme. All rights reserved. Thieme Medical Publishers, Inc., 333 Seventh Avenue, 18th Floor, New York, NY 10001, USA
DOI https://doi.org/ 10.1055/s-0041-1725131. ISSN 0736-6825. 


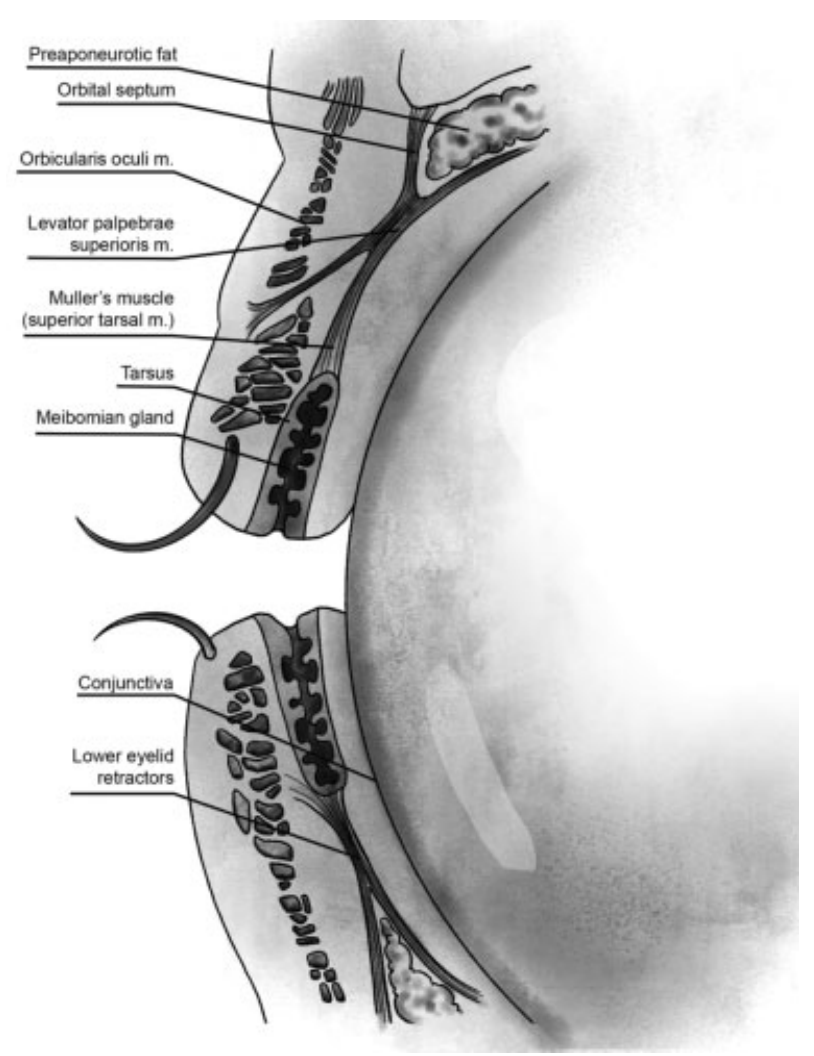

Fig. 1 Sagittal view, anatomy of upper and lower eyelids. Image courtesy, Vivian Hsiao, MD.

anatomical origination of the capsulopalpebral fascia off the inferior rectus muscle and its involvement as it splits and encapsulates the inferior oblique.

The levator muscle originates from the inferior aspect of the lesser wing of the sphenoid and inserts into the superior tarsus and eyelid skin as a thinned, fibrous aponeurosis. The insertion of the aponeurosis into the eyelid skin forms the eyelid crease. An important anatomical landmark in the course of the levator is Whitnall's ligament, also known as the superior transverse ligament, which extends from the lacrimal gland fascia temporally to the trochlea medially. This ligament helps suspend and support the superior orbital soft tissues. It is helpful to think of this ligament as a clothesline over which the levator aponeurosis is draped which helps the levator pull the eyelid superiorly rather than posteriorly. ${ }^{4}$ The analogue of Whitnall's ligament in the lower eyelid is Lockwood's ligament, also known as the suspensory ligament of the eyeball.

The orbicularis oculi muscle is the primary muscle responsible for eyelid closure. It is present in both upper and lower eyelids and can be anatomically divided into three components: the pretarsal, preseptal, and orbital portions, in order moving radially outward from the eyelid margin. As the name suggests, the pretarsal and preseptal orbicularis overlie the tarsus and orbital septum, respectively, underneath. The orbicularis is innervated by the facial nerve from the underneath throughout and any damage here, whether from trauma or iatrogenic, may lead to ectropion or lagophthalmos. ${ }^{5}$

The tarsal plates are structures composed of dense connective tissue which provides structural integrity and sup- port for the eyelids. They abut the eyelid margin; the meibomian glands which secrete oils at the eyelid margin are located within the tarsus. The superior tarsus extends approximately 10 to $12 \mathrm{~mm}$ from the margin, while the lower tarsus is typically 4 to $5 \mathrm{~mm}$ in length. Both tarsal plates extend across most of the length of the eyelid, approximately $29 \mathrm{~mm}$, and taper as they approach the medial and lateral canthi. $^{6}$

The orbital septum is a thin, fibrous plane that extends from the distal edge of the tarsal plates to the orbital rim. The septum acts as a physiological barrier separating intraorbital contents from the orbicularis, skin, and superficial soft tissues. It is of significant importance, for example, in differentiating preseptal cellulitis from orbital cellulitis, as it acts as a barrier to prevent the spread of infection.

Deep to the septum lies the preaponeurotic orbital fat. The upper eyelid preaponeurotic fat can be separated into two fat pads, the medial and central. In contrast, the lower eyelid has medial, central, and lateral fat pads. It is important to distinguish the lacrimal gland, which sits in the preaponeurotic space superiorly and laterally. Compared with fat, the gland is typically more irregular, better vascularized, and paler in color. As the name suggests, the preaponeurotic fat is superficial to the eyelid retractors, the levator superiorly and the lower eyelid retractors inferiorly. It thus acts as an important surgical landmark in locating the retractors (-Fig. 1).

Imagining a full thickness violation of the eyelid, therefore, different structures will be penetrated depending on the distance from the eyelid margin. Proximally, a fullthickness injury to either eyelid will violate skin, orbicularis, tarsus, and palpebral conjunctiva, in anterior-to-posterior order. The former two layers are collectively considered the anterior lamellae while the latter comprise the posterior lamellae. These are distinct at the eyelid margin, separated by the gray line which sits anterior to the meibomian gland orifices and posterior to the lash line. It is valuable to consider these entities separately in understanding the anatomical and functional reconstruction of the eyelids. For example, a superficial wound may leave the posterior lamellae intact and require only closure of the anterior lamellae. On the other hand, a deep burn injury may cause scarring and foreshortening of the posterior lamellae, creating involutional mechanical forces that can cause entropion, trichiasis, and potentially significant discomfort and damage to vision. Distally, a full-thickness injury of the eyelid causes significantly more damage. In addition to skin and orbicularis, then penetrating tarsus, the wound will involve orbital septum, preaponeurotic fat, levator, conjunctiva, and potentially Muller's muscle. Although physiological aging changes cause thinning of the septum and occasionally prolapse of the preaponeurotic fat, the presence of fat in a traumatic wound indicates violation of the septum and should prompt high suspicion for intraorbital injury.

Traumatic injury to the eyelid can be further complicated by involvement of the lacrimal drainage apparatus, which consists of the puncta, canaliculi, lacrimal sac, and nasolacrimal duct. Tears on the surface of the eye first travel 
through the puncta, which sit on a slight elevation of the eyelid called the lacrimal papilla and travel vertically approximately $2 \mathrm{~mm}$ before turning 90 degrees at a dilation known as the ampulla before extending horizontally through the canaliculi. This anatomy is mirrored in both upper and lower eyelids. Both canaliculi course independently for approximately 8 to $10 \mathrm{~mm}$ before fusing nasally to form the common canaliculus which then enters the lacrimal sac at the common internal punctum. ${ }^{7}$ The lacrimal sac sits in the lacrimal sac fossa, a bony pouch formed within the anterior and posterior lacrimal crests. The lacrimal sac is approximately $2 \mathrm{~mm}$ wide and 10 to $15 \mathrm{~mm}$ long vertically, and it tapers inferiorly to form the nasolacrimal duct which then empties tears into the nose in the inferior meatus just below the inferior turbinate (-Fig. 2).

The canthal tendons are responsible for anchoring the eyelids to the lateral and medial orbital rim. The lateral canthal tendon attaches to the lateral orbital rim at a bony prominence called Whitnall's tubercle. The medial canthal tendon forms anterior and posterior limbs that attach to the anterior and posterior lacrimal crests, respectively, and surrounds the lacrimal sac. The lateral canthal tendon is normally approximately $2 \mathrm{~mm}$ superior to the medial canthal tendon; this gives the eyes the characteristic downward slant toward the nose which assists in physiological tear drainage.

\section{Approach to Periocular Trauma}

The initial evaluation of the patient with periocular soft tissue trauma must begin with a holistic assessment of the patient and the inciting traumatic event. The patient's hemodynamic status and cardiopulmonary stability must be

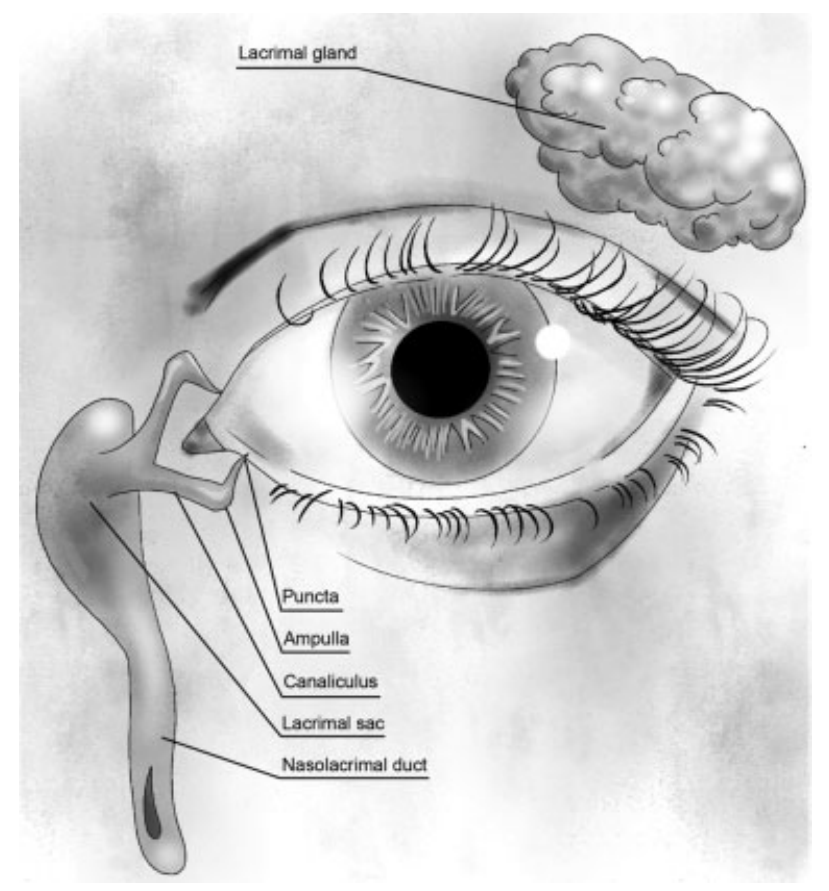

Fig. 2 Anatomy of lacrimal drainage system. Image courtesy, Vivian Hsiao, MD. assured by the primary or trauma team prior to evaluation by the ophthalmologist. Before examining the patient, a thorough history should be taken with the patient or an informed representative. Children may need to be interviewed separately from their guardian(s) to elicit a truthful history. It is important to identify the mechanism of injury, as this may inform clinical suspicion for occult injuries not otherwise immediately evident on exam. The past medical history and time of last oral intake is crucial when anticipating any need for sedation or anesthesia. In addition, it is important to assess for previous surgeries that may distort normal anatomy and to check vaccination status if tetanus is a concern.

Ophthalmic examination must first rule out vision-threatening injury to the orbital socket or globe, which is always antecedent to the repair of any periorbital soft tissues. The vital signs of the eye-visual acuity, relative afferent pupillary defects, and intraocular pressure-should always be measured. Photographic documentation should be acquired before any effort is made to clean or prepare the patient for even basic examination. These may oftentimes be redundant but can prove priceless if the medicolegal need arises. A comprehensive eye exam should rule out the presence of any acute intraocular pathology, such as hyphema (blood in the anterior chamber of the eye), angle recession, lens subluxation, vitreous hemorrhages, or retinal detachments. Most emergently, an intraocular foreign body or a ruptured globe are indications for immediate surgical intervention, and further examination of the periocular soft tissues is unnecessary and indeed oftentimes contraindicated. $^{8}$

Motility and position of the globe are often involved in acute ophthalmic traumas and it may be difficult to differentiate between restrictions caused by soft tissue swelling versus those secondary to bony fractures. The presence of step deformities or crepitus may indicate the latter. Neurological assessment including assessment for facial hypoesthesia and hypokinesia is also important to rule out neurovascular injury. For example, an otherwise asymptomatic patient with new cheek numbness may have an occult orbital floor fracture. When in doubt, noncontrast computed tomography is the preferred imaging modality for assessing the acute trauma patient. While many clinicians develop a personalized workflow, it is helpful to approach these disparate components of the exam in a systematic fashion to ensure efficient, thorough care.

Once the globe has been addressed, attention should be focused on the eyelids. In no particular order, the integrity of the lid margin, anterior and posterior lamellae, puncta and canaliculi, eyelid movement, and periorbital fat should all be noted (-Fig. 2). These should be assessed thoroughly in all patients; as we will discuss more later, even remote injuries such as a punch to the cheek may cause avulsions of the canaliculi and lead to significant functional impairment if not detected and repaired promptly. ${ }^{9}$

\section{Approach to the Eyelid}

Although nondisjointed traumas, such as contusions and abrasions, are typically nonsurgical and may be treated 


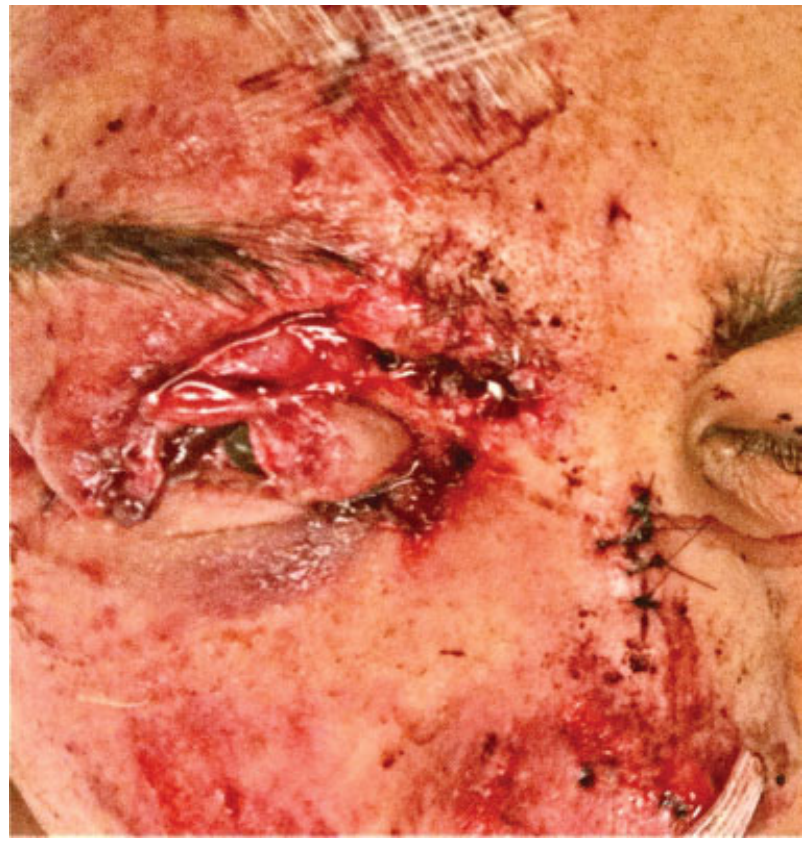

Fig. 3 Pedestrian hit by motor vehicle and dragged sustaining full thickness upper lid and brow damage.

medically and left to heal by secondary intention, it may still be important to undergo procedural repair if the affected areas are cosmetically sensitive, or if cicatricial changes have a significant possibility of functional impairment. ${ }^{8}$ Simple lacerations without missing tissue may be partial thickness or full thickness and lid margin-involving or not. It is important in all kinds of eyelid lacerations to reapproximate both the anterior and posterior lamellae separately. If the lid margin is involved, the gray line and tarsal plate must be adequately opposed to prevent lid notching ( - Figs. 3,4 ). This is typically done with a suture through the meibomian gland orifices; some surgeons may prefer to add an additional suture anteriorly in the lid margin to align the eyelashes. The lid margin should be approximated first prior to reconstruction of the remainder of the eyelid (-Figs. $\mathbf{5}, \mathbf{6}$ ). The suture may be kept long to provide traction for repair and, if nonabsorbable suture is used, such as $6-0$ silk, the tail may be later incorporated into the skin closure to prevent skin irritation and possible corneal abrasions (-Figs. 7, 8). For all repairs, lidocaine with epinephrine is helpful for local anesthesia and vasoconstriction to reduce bleeding but should be used judiciously as extra volume may distort the anatomy and complicate the procedure.

If there is a significant loss of tissue, a larger repair or secondary reconstruction may be indicated. Although older patients with age-related changes often have sufficient excess skin to allow for local advancement flaps, with large defects or patients who are young or otherwise have tight skin, full thickness skin grafts may be necessary to reconstruct the anterior lamellae. Suitable sites for skin donation to replicate eyelid include skin from a different eyelid, preauricular skin, postauricular skin, and supraclavicular skin or that from the underside of the arm. Of note, harvest-

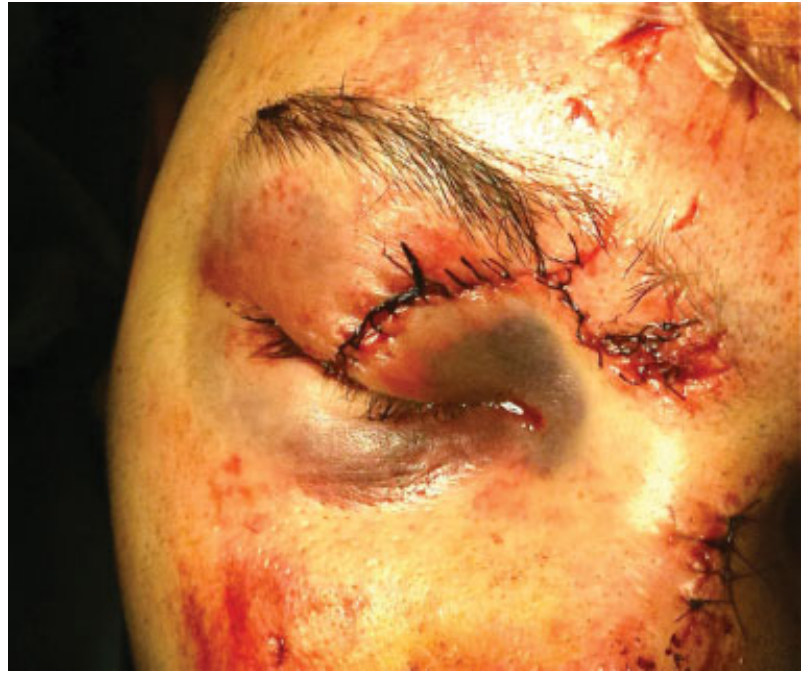

Fig. 4 Repaired upper eyelid and brow laceration. Note that vertical orientation of the reapproximated skin is preferable as this will heal by contracture horizontally and minimize vertical tension forces on the eyelid.

ing skin grafts from the upper eyelid may exacerbate lagophthalmos or cause asymmetry and poor cosmesis, so this option may be less preferred compared with the alternative donor sites.

It is generally preferred to reconstruct the posterior lamellae using a flap rather than a graft, as this provides better vascular supply for wound healing and better reconstructs the fibrous structure of the tarsus. If primary posterior lamellar closure is not immediately possible, a simple canthotomy and cantholysis may provide sufficient lateral

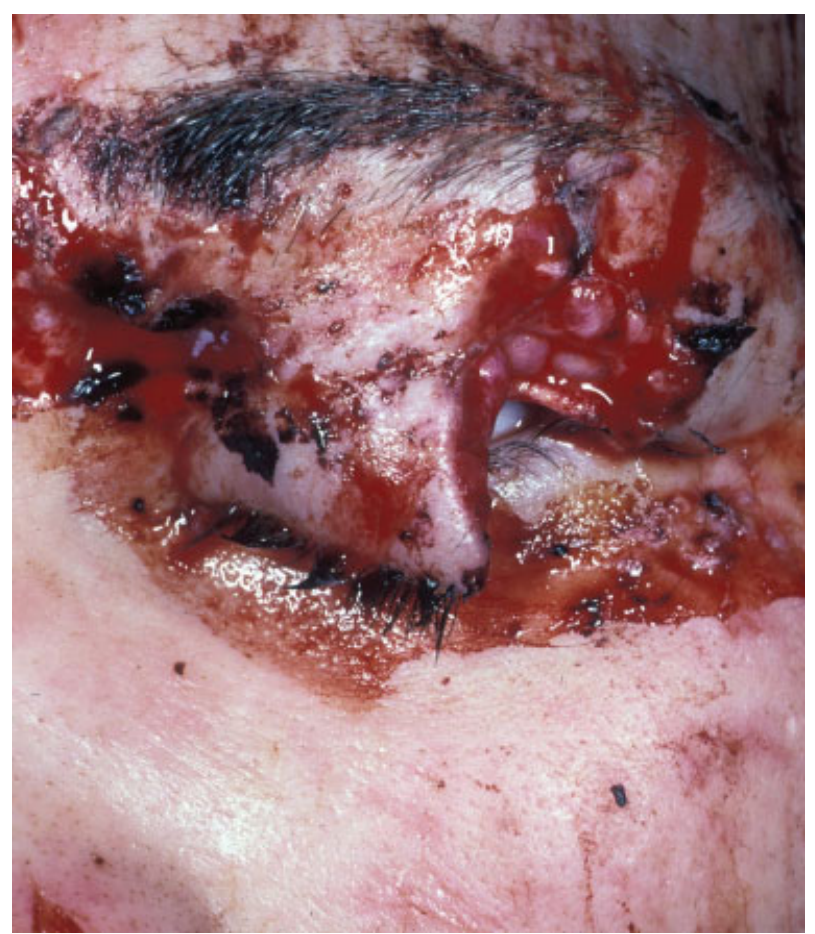

Fig. 5 Motorcycle accident, patient not wearing a helmet, sustaining full thickness upper lid injury with no tissue loss. 


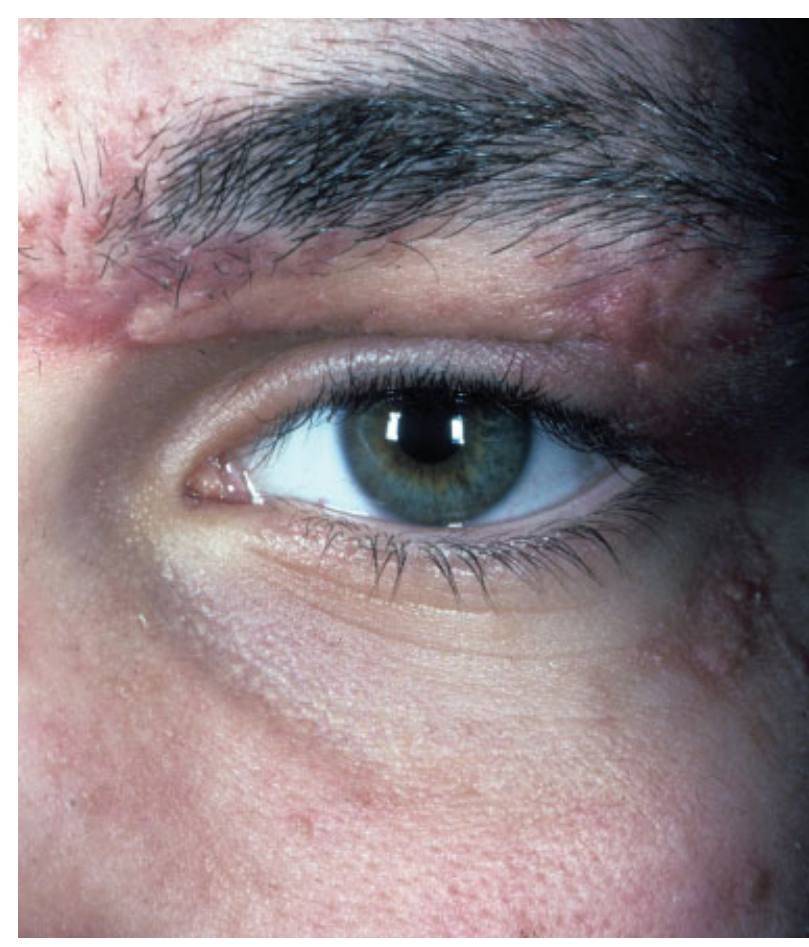

Fig. 6 Three months postoperative repair of upper lid lacerations.

laxity to enable primary closure. Otherwise, a semicircular flap, also called the Tenzel flap, may be utilized whereby the lateral canthotomy is extended superiorly and laterally in a semicircular fashion and advanced medially. Larger defects are common following excisions of malignancies but are relatively rare in traumatic scenarios. If the semicircular flap is insufficient still, a tarsoconjunctival flap, also known

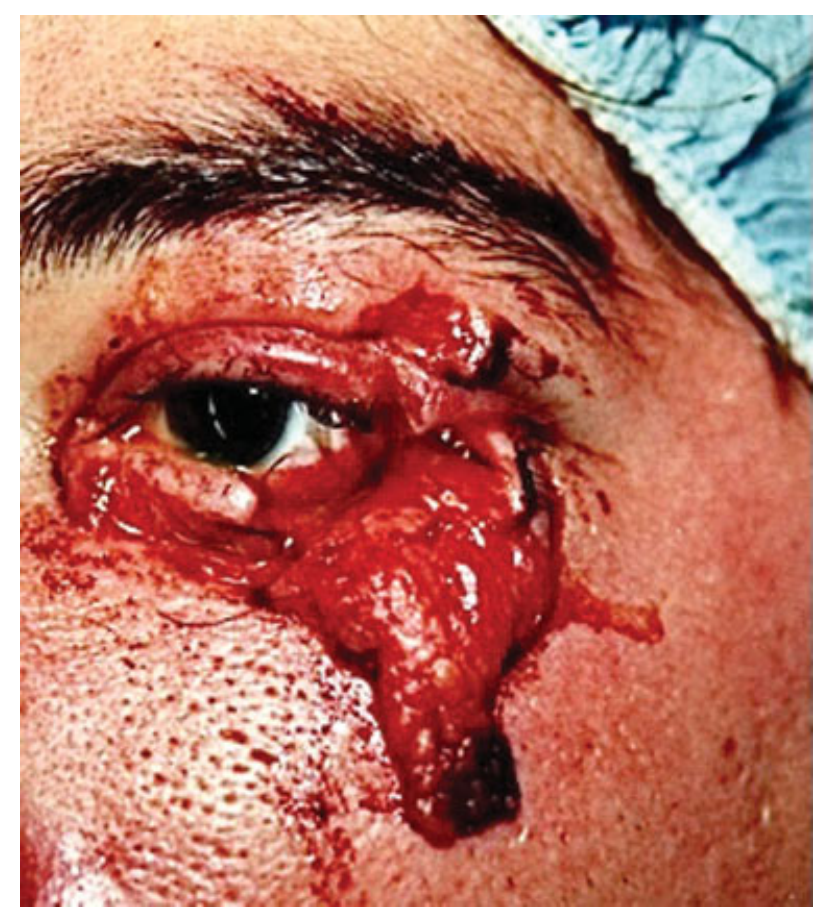

Fig. 7 Young man assaulted with severe full thickness lower lid injury, moderate upper lid laceration.

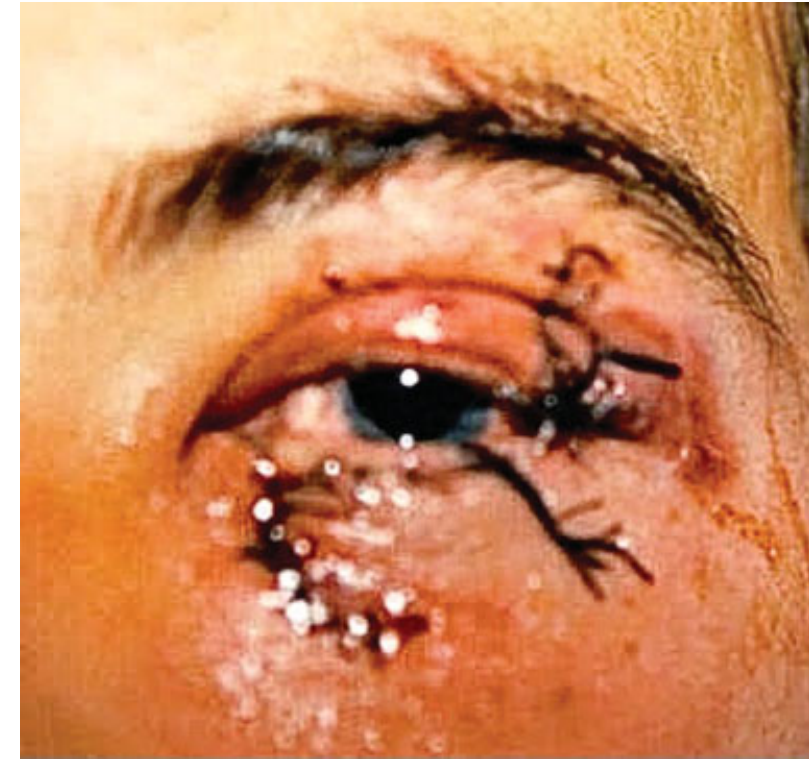

Fig. 8 Repaired lacerations of both the upper and lower eyelids.

as the Hughes flap, may be used. ${ }^{8}$ In this procedure, the posterior lamellae from the upper eyelid is utilized to close a large defect in the lower eyelid. The eye remains closed while the upper eyelid is sutured into the lower eyelid wound in this lid-sharing surgery. A second procedure is then required in 3 to 4 weeks to incise the flap and allow the eye to open.

As a general principle, deep sutures should be absorbable, while nonabsorbable sutures are preferred for superficial closures as these minimize inflammation and reduce unpredictability in the healing process, such as hyperkeratosis or hyperpigmentation. ${ }^{8}$ The patient should utilize antibiotic ointment regularly for lubrication and infection prophylaxis, and the vision should be monitored closely throughout followups to endure uncomplicated healing. Sutures should be left in place for 1 to 2 weeks prior to removal (-Figs. 7-12).

\section{Canalicular Injuries}

The lacrimal drainage apparatus should be of particular concern when evaluating eyelid trauma, with up to $16 \%$ of all eye injuries involving the tear drainage system. ${ }^{10}$ Any eyelid trauma medial to the puncta involves the canaliculus until proven otherwise. Such facial traumas are common in a bimodal distribution. Younger patients more commonly present with sequelae of fistfights, animal bites, or intoxication. These patients are also more often male than female, with studies suggesting a predominance of $2: 1$ to $4: 1 .^{11-13}$ Blouse hooks are a notable cause of canalicular lacerations in breastfeeding infants. ${ }^{14}$ Older patients often present with fall injuries. Middle-age patients have the lowest incidence of canaliculus-involving traumatic injuries. The upper and lower puncta contribute relatively equal amounts to tear drainage, and late-presenting injuries may present with epiphora rather than acute trauma secondary to scarring and malunion of occultly damaged canalicular structures. ${ }^{15,16}$

Special consideration should be given to dog bites, which have a significantly increased risk of canalicular involvement 


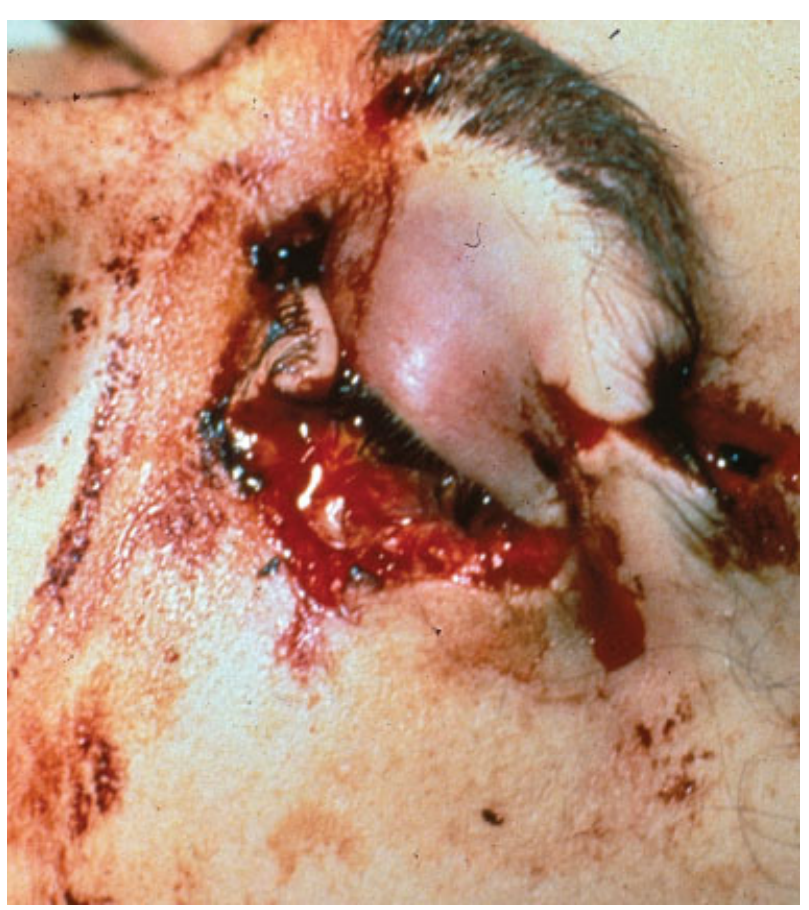

Fig. 9 Victim of knife assault where the blade broke off in her head

and present unique challenges compared with other forms of direct, penetrating trauma. Dogs are the most common source of animal bites and disproportionately affect the face, head, and neck in children, with $35 \%$ or more of eyelid-involving bites also involving the lacrimal drainage apparatus. ${ }^{17-19}$ Bite and nonbite traumas similarly affect predominantly male patients and mostly involve the inferior

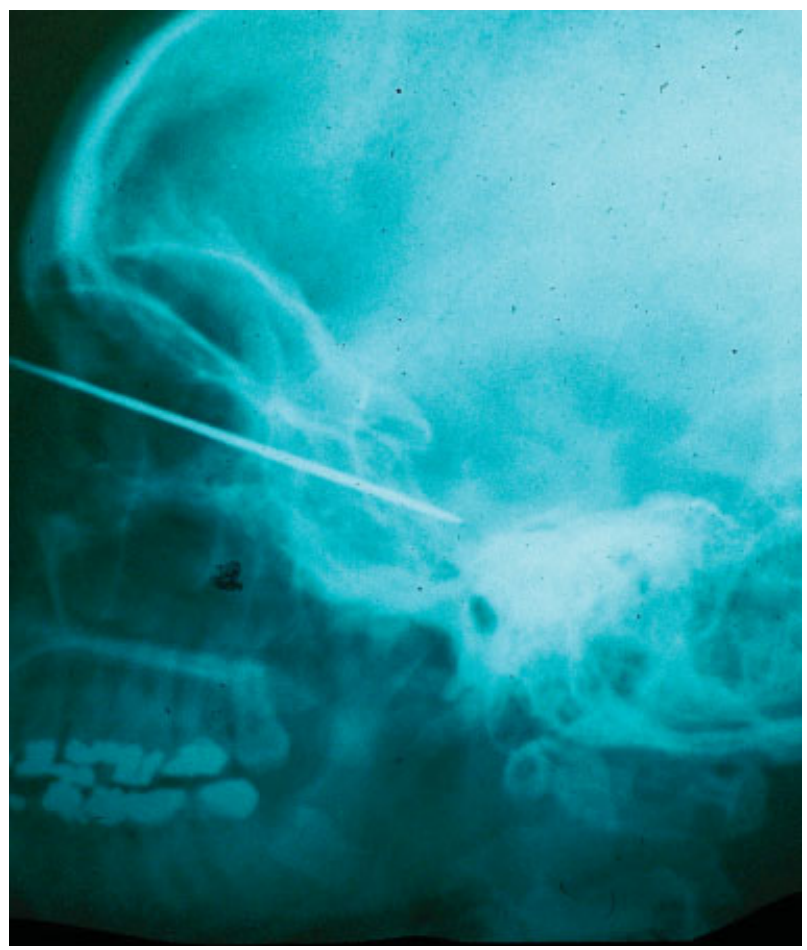

Fig. 10 Sagittal CT scan of knife blade going through orbit.

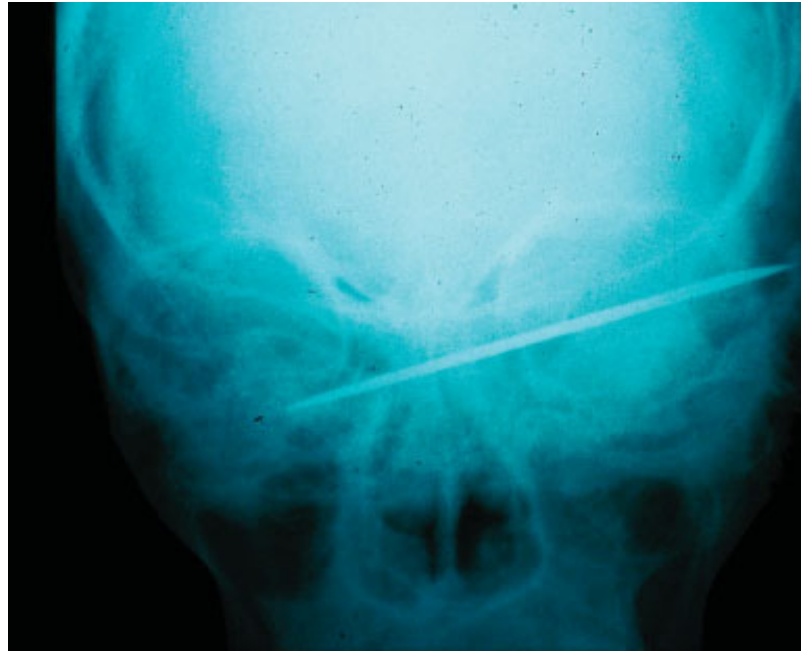

Fig. 11 Coronal CT scan of broken knife blade in orbit.

canaliculi. However, bite traumas require significantly more complicated repairs and necessitate longer follow-up. Wound infections are typically multibacterial, and broadspectrum antibiotics targeted at common canine oral flora in addition to human skin flora should be administered. ${ }^{18,20}$ Most commonly, this may be treated with a combination of $\beta$-lactam antibiotic and $\beta$-lactamase inhibitor, although alternative choices such as clindamycin and a fluoroquinolone or a second generation cephalosporin with anaerobic activity are reasonable in patients with allergies. ${ }^{21}$ Other rare, but life-threatening considerations include assessing the need for tetanus or rabies prophylaxis and evaluating for immunosuppression. Capnocytophaga canimorsus is rare but is a particularly morbid infection following dog bites that may cause endocarditis, meningitis, sepsis, and death, with mortality rates up to $30 \%$ even in immunocompetent patients. 22,23

Trauma to the canaliculus can be broadly categorized into direct and indirect injuries. ${ }^{9}$ Direct lacerations to the canaliculus often occur near the puncta (-Fig. 13), whereas indirect injuries occur on the basis of diffuse trauma or by avulsion from a distant (e.g., lateral canthal) trauma. These

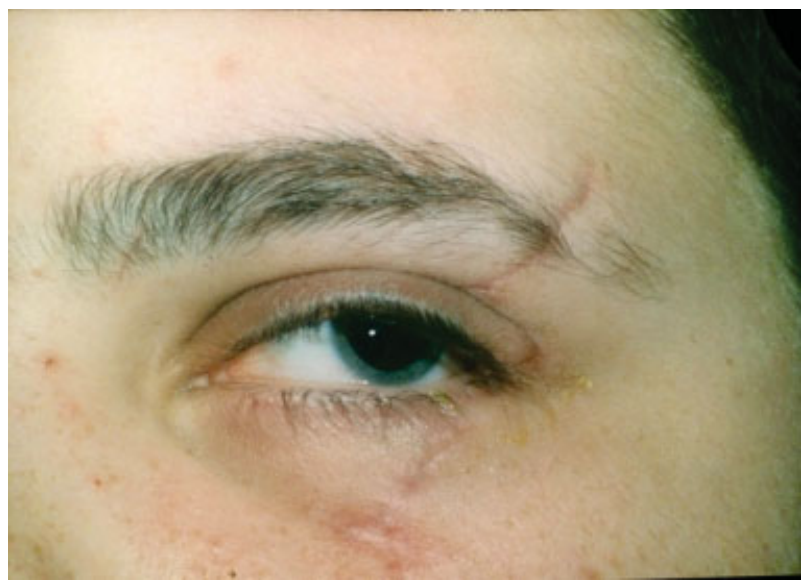

Fig. 12 Patient 4 months postoperative. 


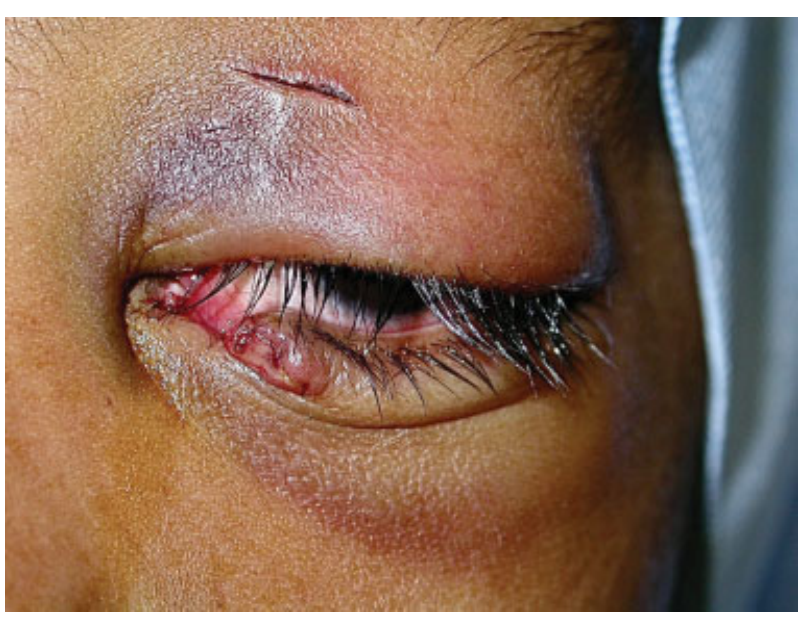

Fig. 13 Lower lid canalicular laceration.

indirect injuries typically result in stretching of the canaliculi and cause tearing deeper near its insertion into the lacrimal sac. Indirect injuries are also more commonly associated with extensive injuries elsewhere in the orbit. Although these etiologies vary significantly depending on geographic location and acute care setting, indirect injuries appear to be more common, ${ }^{13,24,25}$ although one large retrospective review also found that direct injuries occur with comparable frequency. ${ }^{26}$

When there is a suspicion for canalicular injury, it is important to identify any canalicular dehiscence precisely for repair (-Figs. 13,14). One may consider probing wherein a long, thin Bowman probe is introduced perpendicularly to the puncta of the eyelid under suspicion, advanced to a depth of $2 \mathrm{~mm}$ to the ampulla, before being rotated 90 degrees to be parallel to the eyelid margin and further advanced to the length of the canaliculus (-Fig. 15). The probe should advance easily through an untraumatized canaliculus until resistance is felt at the nasal side wall. If the canaliculus is violated, the probe should be visible externally as it protrudes at the site of trauma. Young children may require examination under anesthesia for adequate examination or repair.

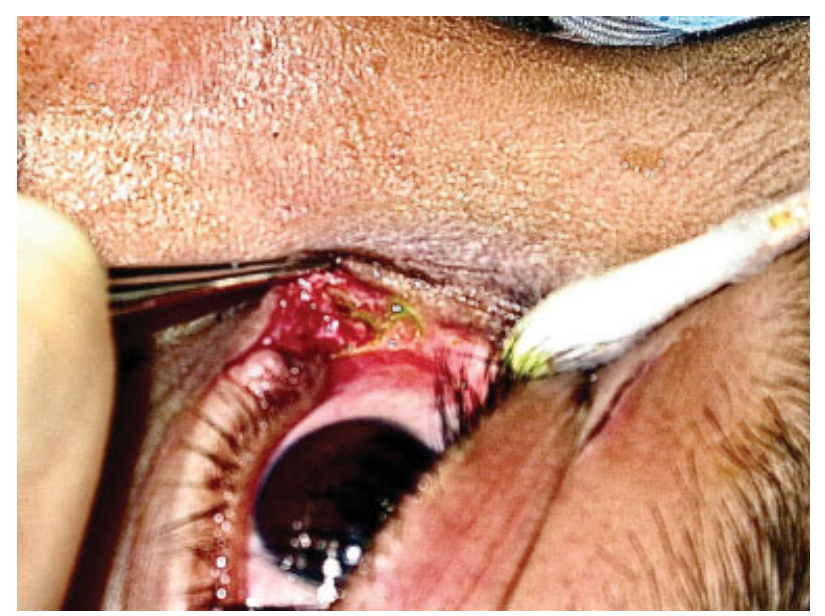

Fig. 14 Using Fluorescein dye to identify the cut ends of the canaliculus.

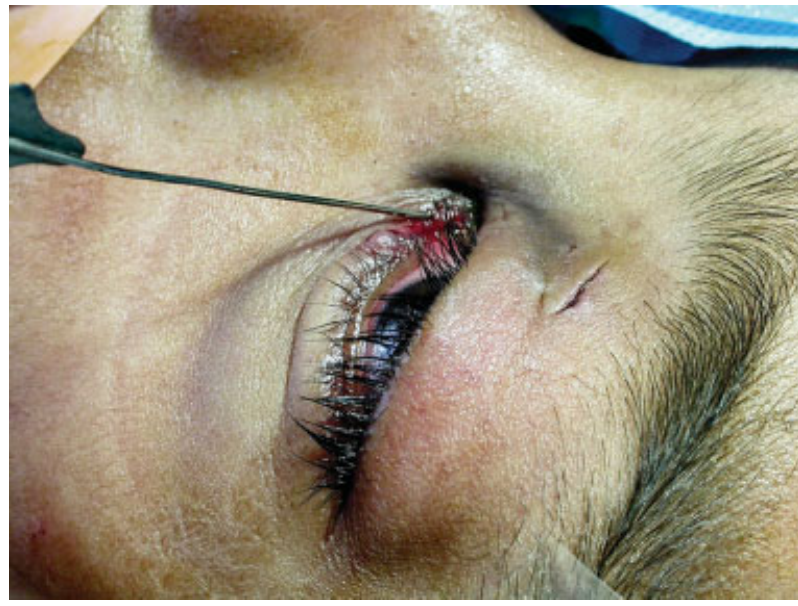

Fig. 15 A lacrimal probe being used to identify the nasal end of a canalicular laceration. Note how the probe is being held nearly horizontally, parallel to the eyelid margin, to follow the physiological anatomical course of the canaliculi.

Trauma to the canaliculus should be repaired whenever possible. Although there may be concomitant injuries of higher priority, delay of canalicular repair, especially beyond 24 to 48 hours, has shown an increased risk of complications. ${ }^{7,12,27}$ These complications include both poor anatomical and functional results, such as malposition of the puncta or nonunion of the canaliculus, as well as ongoing epiphora despite patent anatomy as confirmed by probing and irrigation.

Although a vital diagnostic tool in the diagnostic armamentarium, probing may further traumatize the canaliculus and may complicate repair. A variety of alternative techniques have been developed to assist with atraumatic localization of canalicular injuries. Simple irrigation of water into the puncta with a blunt cannula does not require significant advancement into the canalicular system; a patent lacrimal drainage apparatus should facilitate the patient to be able to taste the injected material. Occasionally, irrigation enables direct visualization of the canalicular laceration, but leakage from the wounded canaliculus may also be difficult to distinguish from reflux from the puncta.

Alternative techniques to assist in localization include injection of fluorescein (-Figs. 14, 15) or air into the puncta and looking for the characteristic leakage of dye or bubbles at the site of trauma. ${ }^{28}$ Topical phenylephrine may be utilized to induce local vasoconstriction and reduce bleeding that may obscure acute traumatic injuries. A more novel technique is to utilize viscoelastic to thoroughly coat the area under suspicion. ${ }^{29}$ This can assist in tamponade and retraction of the affected area while simultaneously helping to dilate the affected canaliculus, facilitating further repair.

There are three major techniques by which canalicular lacerations may be repaired. Common to all of them is the need for stenting of the canaliculus to ensure patency as the lid heals. Bicanalicular stents are placed into both upper and lower lid puncta and are the preferred modality even when there is injury to only one due to greater flexibility and durability (-Fig. 16). Bicanalicular (Crawford) stents may be 


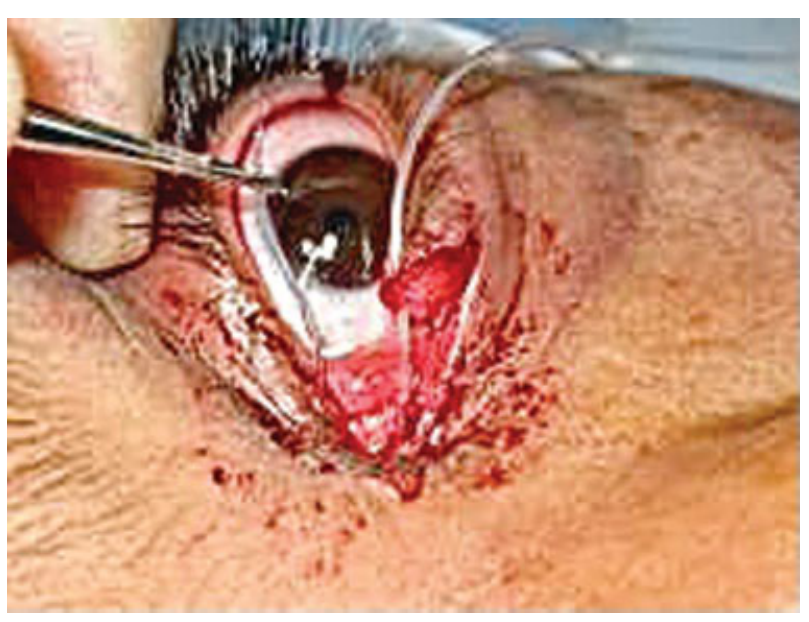

Fig. 16 Using a silicone tube to intubate the canaliculi.

introduced either with nasolacrimal duct intubation or local to the canaliculi only using a pigtail probe. A Crawford stent consists of silicone tubing with double-ended metallic probes which are inserted into the nasal cavity via the canaliculi. A pigtail probe has a circular metal tip that can be introduced to the unaffected canaliculus, through the common canaliculus, and then exposed in the nasal portion of the lacerated canaliculus. ${ }^{30}$ A suture is then passed through the eye of the probe, which is withdrawn, and the stent is then passed over the suture to intubate the canaliculi (-Fig. 17). The lateral portion of the canaliculus can be intubated in a similar fashion.

Compared with pigtail probing, Crawford stenting is more robust and facile when operative repair is otherwise indicated for laceration repair but is disadvantageous in being prohibitively difficult to perform under local anesthesia alone due to the intranasal component. As an alternative to both options, monocanalicular stents may also be used for lacerations limited to a single canaliculus. These have the advantage of being less invasive in placement but may be

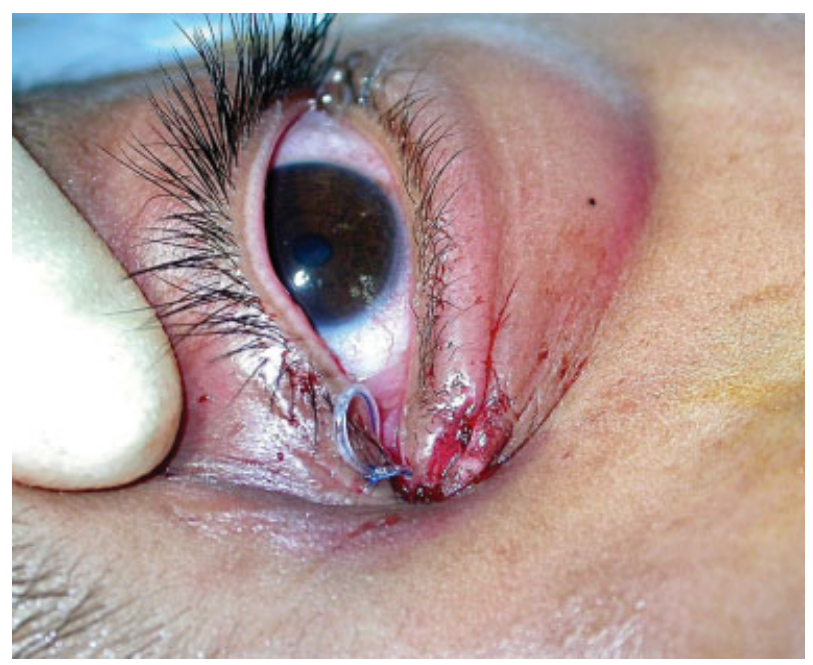

Fig. 17 A bicanalicular stent intubating both upper and lower puncta. This much slack, if left as is, would be at high risk for tube extrusion. It would be further reduced into the interpalpebral space by the completion of the soft tissue repair. commensurately at higher risk for tube extrusion or nonunion.

As a general principle, once the stent is in place, it is preferred to have direct closure of the canaliculus by reanastomosing the cut ends. ${ }^{7,11}$ However, newer innovations in techniques, such as applying lateral traction with the Crawford tube, may reduce the need for doing so without increasing a risk of complications. ${ }^{24}$ Superficial lacerations involving the canaliculus may then be repaired with simple closure of skin overlying the stent. For deeper lacerations or avulsed wounds involving the medial canthal tendon, this should be reconstructed with a traction suture reattaching the posterior reflection of the tendon to its anatomical position at the periosteum of the posterior lacrimal crest. This is important both to relieve tension on the healing canaliculus and to support the eyelid and prevent ectropion. Surgical repair of the posterior medial canthal tendon may occasionally be difficult due to the lacrimal sac, and limited retrospective reviews have suggested bicanalicular stenting alone may be sufficient in cases without anterior involvement or medial canthal tendon displacement. ${ }^{11,31}$

Following reconstruction, antibiotic ointment should be applied to the site of repair. Patients may be followed postoperatively initially at 1 to 2 weeks interval, gradually extending to 1 to 2 months follow-up with progressive normal healing. Stents are most commonly removed after 3 months, although this may be safely extended to a period of 4 to 6 months depending on the extent of the trauma and subsequent repair. ${ }^{11,13,24}$ Crawford stents may be removed from within the nares with cooperation from the patient if the stent can be located and cut above the knot. If the stent cannot be visualized endonasally, it may also be removed from the puncta. Following the instillation of topical anesthetic, the stent should be rotated to visualize the knot, which should then be cut and pulled in a manner to avoid pulling the loose end with the knot through the canalicular system. Stents from pigtail probes may be removed in a similar fashion. Monocanalicular stents may simply be removed from the puncta. These are often held in place by a dilated plug on one end and dislodge with gentle traction. Some surgeons may prefer to suture these in place, in which case this should be cut prior to stent removal.

\section{Eyelid Burns}

We will take a moment to note that thermal burn injuries are beyond the scope of this review but commonly involve the eyelid. Treatment of burns is very complex and must address the initial inflammatory cascade and significant edema that develops, which can cause orbital compartment syndrome in severe cases, as well as intermediate sequelae such as exposure or infection and late cicatricial complications such as ectropion, retraction, forniceal foreshortening, and canalicular stenosis. These often require close monitoring and multiple interventions such as debridement and grafting to treat. $^{32}$

However, the same principles established above inform the clinician's priorities in treating periocular burn injuries. 
Life-threatening injuries must first be ruled out or otherwise stabilized. Expedient consultation with the burn team is necessary to optimize the patient's outcomes. The periocular skin, as discussed previously, is thin and is devoid of subcutaneous fat which might protect against full thickness extension of burns. The globe must be protected to prevent vision-threatening injuries, and tarsorrhaphy may be used to temporarily or permanently close a portion of the eyelids to this effect. The eyelid margin and canaliculus must be evaluated and reconstructed separately, although the latter is deeper and rarely directly involved in burns.

\section{Conclusion}

Eyelid skin is the thinnest in the body, which is anatomically necessary to facilitate the frequent movement associated with blinking. ${ }^{33}$ The minute details of the anatomy hidden therein pose unique reconstructive challenges in achieving the ideal functional outcome. Approaching the eyelid as the basic building blocks of anterior and posterior lamellae, eyelid margin, and canaliculus as separate targets for repair helps provide a guideline to understand and perform oculoplastic repair of eyelid traumas. In the acute setting, prompt restoration of these components is important to enable healing and protect vision. This reduces the risk of later sequelae such as ectropion, epiphora, or ptosis which can also be surgically managed in a controlled secondary operation after the acute inflammation has resolved.

Patients should also be educated on wound care, such as the avoidance of eye rubbing, to optimize the healing process. If a secondary procedure or operation is anticipated, such as stent removal in several months, patients should be counseled to expect this to reduce the risk of being lost to follow-up. Patients should also be instructed on postoperative expectations, such as physiologic tearing while a stent is in place; this collaborative, joint decision-making process can improve the patient's prognosis and their perception of their repair. When indicated, early referral to other subspecialists, such as plastic surgeons or oral maxillofacial surgeons, is helpful to create a holistic, broad-based approach in the treatment of eyelid traumas.

Conflict of Interest

None declared.

\section{References}

1 Abdelhady A, Patel BC. Anatomy, Head and Neck, Eye Superior Tarsal Muscle (Mullers Muscle). Treasure Island, FL: StatPearls; 2020

2 Esperidião-Antonio V, Conceição-Silva F, De-Ary-Pires B, PiresNeto MA, de Ary-Pires R. The human superior tarsal muscle (Müller's muscle): a morphological classification with surgical correlations. Anat Sci Int 2010;85(01):1-7

3 Baldwin HC, Bhagey J, Khooshabeh R. Open sky Müller muscleconjunctival resection in phenylephrine test-negative blepharoptosis patients. Ophthal Plast Reconstr Surg 2005;21(04): 276-280

4 Codère F, Tucker NA, Renaldi B. The anatomy of Whitnall ligament. Ophthalmology 1995;102(12):2016-2019
5 Gordin E, Lee TS, Ducic Y, Arnaoutakis D. Facial nerve trauma: evaluation and considerations in management. Craniomaxillofac Trauma Reconstr 2015;8(01):1-13

6 Cochran ML, Lopez MJ, Czyz CN. Anatomy, Head and Neck, Eyelid. Treasure Island, FL: StatPearls; 2020

7 Reifler DM. Management of canalicular laceration. Surv Ophthalmol 1991;36(02):113-132

8 Ko AC, Satterfield KR, Korn BS, Kikkawa DO. Eyelid and periorbital soft tissue trauma. Facial Plast Surg Clin North Am 2017;25(04): 605-616

9 Wulc AE, Arterberry JF. The pathogenesis of canalicular laceration. Ophthalmology 1991;98(08):1243-1249

10 Herzum H, Holle P, Hintschich C. Eyelid injuries: epidemiological aspects. Ophthalmologe 2001;98(11):1079-1082

11 Tint NL, Alexander P, Cook AE, Leatherbarrow B. Eyelid avulsion repair with bi-canalicular silicone stenting without medial canthal tendon reconstruction. $\mathrm{Br} J$ Ophthalmol 2011;95(10):1389-1392

12 Raj A, Thakur S, Arya KS, Kesarwani P, Sinha U. Canalicular lacerations in a tertiary eye hospital: our experience with monocanalicular stents. Rom J Ophthalmol 2020;64(02):146152

13 Guo T, Qin X, Wang H, et al. Eiology and prognosis of canalicular laceration repair using canalicular anastomosis combined with bicanalicular stent intubation. BMC Ophthalmol 2020;20(01): 246

14 Naik MN, Kelapure A, Rath S, Honavar SG. Management of canalicular lacerations: epidemiological aspects and experience with Mini-Monoka monocanalicular stent. Am J Ophthalmol 2008;145(02):375-380

15 Linberg JV, Moore CA. Symptoms of canalicular obstruction. Ophthalmology 1988;95(08):1077-1079

16 Daubert J, Nik N, Chandeyssoun PA, el-Choufi L. Tear flow analysis through the upper and lower systems. Ophthal Plast Reconstr Surg 1990;6(03):193-196

17 Sadiq MA, Corkin F, Mantagos IS. Eyelid lacerations due to dog bite in children. J Pediatr Ophthalmol Strabismus 2015;52(06): 360-363

18 Savar A, Kirszrot J, Rubin PA. Canalicular involvement in dog bite related eyelid lacerations. Ophthal Plast Reconstr Surg 2008;24 (04):296-298

19 Slonim CB. Dog bite-induced canalicular lacerations: a review of 17 cases. Ophthal Plast Reconstr Surg 1996;12(03):218222

20 Botek AA, Goldberg SH. Management of eyelid dog bites. J Craniomaxillofac Trauma 1995;1(02):18-24

21 Talan DA, Citron DM, Abrahamian FM, Moran GJ, Goldstein EJEmergency Medicine Animal Bite Infection Study Group. Bacteriologic analysis of infected dog and cat bites. N Engl J Med 1999;340(02):85-92

22 Mader N, Lührs F, Langenbeck M, Herget-Rosenthal S. Capnocytophaga canimorsus-a potent pathogen in immunocompetent humans-systematic review and retrospective observational study of case reports. Infect Dis (Lond) 2020;52(02):65-74

23 Zajkowska J, Król M, Falkowski D, Syed N, Kamieńska A. Capnocytophaga canimorsus-an underestimated danger after dog or cat bite-review of literature. Przegl Epidemiol 2016;70(02): 289-295

24 Baek SO, Kim JW, Lee JY, Kwon H. Traction applying technique on bicanalicular Crawford silicone tube to overcome the notching deformity after lacrimal canalicular repair. J Craniofac Surg 2020 (e-pub ahead of print). Doi: 10.1097/scs.0000000000006956

25 Rishor-Olney CR, Hinson JW. Canalicular Laceration. Treasure Island, FL: StatPearls; 2020

26 Jordan DR, Ziai S, Gilberg SM, Mawn LA. Pathogenesis of canalicular lacerations. Ophthal Plast Reconstr Surg 2008;24(05): 394-398

27 Dortzbach RK, Angrist RA. Silicone intubation for lacerated lacrimal canaliculi. Ophthalmic Surg 1985;16(10):639-642 
28 Morrison FD. An aid to repair of lacerated tear ducts. Arch Ophthalmol 1964;72:341-342

29 Örge FH, Dar SA. Canalicular laceration repair using a viscoelastic injection to locate and dilate the proximal torn edge. J AAPOS 2015;19(03):217-219

30 Jordan DR, Nerad JA, Tse DT. The pigtail probe, revisited. Ophthalmology 1990;97(04):512-519
31 Tawfik HA, Elsamkary M. Authors' response: eyelid avulsion repair with bi-canalicular silicone stenting without medial canthal tendon reconstruction. Br J Ophthalmol 2012;96(07):1042-1043

32 Malhotra R, Sheikh I, Dheansa B. The management of eyelid burns. Surv Ophthalmol 2009;54(03):356-371

33 Pe'er J. Pathology of eyelid tumors. Indian J Ophthalmol 2016;64 (03):177-190 